\title{
Estruturas do Sistema de Saúde: do Complexo Médico-industrial ao Médico-financeiro
}

CID MANSO DE MELLO VIANNA ${ }^{1}$

O conceito de complexo médico-industrial tem sido utilizado, desde os anos 1980, no Brasil, para ressaltar as múltiplas e complexas inter-relações estabelecidas entre os diversos atores do setor saúde e destes com os demais setores da economia. O CMI é um produto histórico e particular da evolução do sistema de saúde. É um estágio em que, devido à necessidade de reprodução dos capitais investidos, as práticas capitalistas privadas se tornam hegemônicas e determinantes das funções, papéis e relações de cada ator no interior do próprio sistema. A hipótese discutida neste texto é que o sistema de saúde tem hoje dois atratores fundamentais, moldando o comportamento de seus agentes: um é a tecnologia e o outro é o aspecto financeiro da valorização do capital. Para o primeiro, constituiu-se o que se denomina complexo médico-industrial, enquanto que, para o segundo, tem-se o aparecimento de um complexo médico-financeiro.

Palavras-chave: Sistema de saúde; complexo médico-industrial; tecnologia médica. 
Muitas empresas privadas, além do setor público, atuam na área da saúde - as produtoras de serviços mercantis de saúde; as de origem financeira, como as companhias de seguros de saúde; as produtoras, nacionais ou não, dos diversos insumos e bens de capital utilizados na prestação dos serviços médicos, como medicamentos, materiais e equipamentos médicos e as famílias, beneficiárias finais dos serviços de saúde (mercantis e não-mercantis).

Entender o intercâmbio entre esses agentes, cujos interesses são variados, tem sido objeto de análise de diversos pesquisadores nacionais ${ }^{2}$ e internacionais ${ }^{3}$. Estes têm procurado esclarecer os principais aspectos da lógica de comportamento de cada um dos participantes do setor saúde, embora poucos tenham tentado explicar de maneira mais geral seu funcionamento. Uma das principais explicações é a utilização da idéia de complexo, feita a partir dos anos 1980, sobre a racionalidade econômica e tecnológica interna à saúde.

Esse conceito tem sido utilizado desde a década de 80 no Brasil, com o propósito de ressaltar as múltiplas e complexas inter-relações estabelecidas entre os diversos atores do setor saúde e destes com os demais setores da economia. A proposta era estudar as relações entre o Estado e o complexo médico empresarial, visando a melhor elucidar as origens e a dinâmica contraditória do desenvolvimento das empresas médicas no Brasil e o padrão de consumo dos bens de saúde.

O conceito de complexo médico-industrial (CMI) passou a ser concebido de modo ampliado, contemplando as diferentes articulações entre a assistência médica, as redes de formação profissional, a indústria farmacêutica e a indústria produtora de equipamentos médicos e de instrumentos de diagnóstico ${ }^{4}$. O CMI é um produto histórico e particular da evolução do sistema de saúde. É um estágio onde, devido à necessidade de reprodução dos capitais investidos, as práticas capitalistas privadas tornam-se hegemônicas e determinantes das funções, papéis e relações de cada ator no interior do próprio sistema (Vianna, 1993).

Na literatura econômica, o conceito de complexo tem sido utilizado, com bastante freqüência, na análise das relações e da dinâmica intersetorial da indústria (Pereira, 1985). O foco central na demarcação do conceito de complexo está na idéia de independência ou autonomia de um bloco de setores vis-à-vis o conjunto de indústrias da economia.

Um complexo industrial significa um conjunto de indústrias no qual os vínculos expressos pelos fluxos de bens e serviços entre elas são mais fortes do que as ligações existentes com outros setores da economia. Assim: 
“[...] a idéia chave é de interdependência entre blocos de setores. Resta, a partir desta conceituação mais geral, definir, de um lado, quais são os fluxos econômicos relevantes a serem levados em consideração na análise e, de outro, qual a metodologia mais apropriada para, a partir destas relações e respeitando o princípio geral de definição do conceito, montar o conjunto de complexos" (Pereira, 1985, p. 98).

Todo complexo industrial - e o complexo médico-industrial não constitui uma exceção - traz, em si mesmo, o caráter único das relações que se estabelecem entre seus membros e a forma pela qual cada um deles, em particular, e o complexo, no geral, se apropriam de uma parcela do excedente econômico. Assim, não basta somente analisar os vínculos existentes a partir dos fluxos de bens e serviços, que poderiam ser adequadamente tratados a partir da idéia de cadeias de produção. É preciso também entender o papel que os diversos agentes e elementos constituintes de um complexo industrial assumem na sociedade e como as relações entre eles se processam ao longo do tempo.

Para isto, talvez, seja mais interessante buscar em uma nova disciplina, a Teoria dos Sistemas Complexos ${ }^{5}$, uma proposta explicativa do comportamento dos agentes do setor saúde. Atualmente, essa teoria tem sido utilizada para explicar os mecanismos envolvidos em sistemas distintos, como terremotos, o comportamento de partículas atômicas, a evolução das espécies, o sistema nervoso central e a própria economia.

Sistemas complexos são formados por unidades simples que estão interligadas entre si, de modo que o comportamento de cada uma influencia a das outras e realimenta dinamicamente o próprio sistema. A complexidade é função dessa interdependência, à medida que o sistema se transforma (Oliveira, 1999). Algumas das principais características de sistemas complexos seriam: adaptação, compromissos locais e provisórios, conflitos, multiequilíbrios e atratores.

Em um sistema adaptativo, os agentes mudam constantemente o seu comportamento com o tempo, em função do que aprendem com a experiência. Não significa, ao contrário do que se poderia esperar, aumento da eficiência do sistema. Esse aprendizado não otimiza, não produz o melhor resultado possível. Isto porque as decisões dos diversos atores de um complexo - por exemplo, adotar ou não uma tecnologia específica, escolher um ou outro fornecedor - podem ser locais e provisórias. São gerados, nesse contexto, tantos compromissos como conflitos não permanentes, com o 
aparecimento de vários pontos de equilíbrio metaestáveis, nos quais a possibilidade de um sistema ótimo pode até ser difícil de ser definida, quanto mais encontrada.

Ainda que sistemas complexos adaptativos apresentem a existência de uma complexa interconexão de influências entre seus agentes, o comportamento de cada um não só não é aleatório, mas segue determinados padrões. Normalmente se constituem a partir de uma racionalidade imposta pelos atratores, na qual os agentes tendem para uma situação produto de muitos dos possíveis estados iniciais. É importante distinguir aqui que sistemas complexos podem possuir mais de um atrator e produzir mais de um estágio de equilíbrio.

A hipótese que se discute a seguir é que o sistema de saúde tem hoje dois atratores fundamentais moldando o comportamento de seus agentes. Um é a tecnologia e o outro é o aspecto financeiro da valorização do capital. Para o primeiro, constituiu-se o que se denomina complexo médico-industrial, enquanto que, para o segundo, tem-se o aparecimento de um complexo médico-financeiro.

\section{Complexo Médico-industrial}

A caracterização de um setor produtivo qualquer pode ser feita de várias formas e o conceito de complexo médico-industrial (Cordeiro, 1980; Vianna, 1993), principalmente se atualizado à luz das mudanças sociais, econômicas e setoriais ocorridas nos últimos quinze anos, pode ser uma opção proveitosa para se caracterizar o setor saúde no Brasil.

No caso do complexo da saúde, é necessário entender que a dinâmica e a evolução dos sistemas de saúde dependem de duas racionalidades, uma interna e outra externa ao sistema. A racionalidade interna é definida pela existência de um padrão de acumulação de capital, a partir de duas lógicas distintas - uma pública e outra privada. Já a racionalidade externa compreende o conjunto das demandas sobre o sistema de saúde que influenciam o modus operandi do sistema e de seus agentes.

A conformação e a dinâmica do complexo médico-industrial resultam do próprio processo de desenvolvimento histórico do sistema de saúde, sendo, portanto, um de seus estágios particulares de evolução. Este se constituiu, ao longo do tempo, como uma resposta à função que, de um lado, a medicina tem no mundo moderno e, de outro, ao papel que a tecnologia assume na forma como se estabelecem as relações de produção e as trocas entre os 
componentes do setor, em particular após o advento da revolução tecnológica ocorrida nas indústrias farmacêutica e de equipamentos médicos, após a II Guerra Mundial (Vianna, 1995a e b).

A partir da Segunda Guerra, nos anos 50, a indústria farmacêutica, que emergiu como produto das novas descobertas de medicamentos, passou a influenciar crescentemente a prática médica. Médicos e pacientes tinham agora à sua disposição um quantitativo surpreendente de novas drogas que ofereciam a promessa de resolver "velhos" problemas de saúde. O impacto sobre as condições de saúde dos indivíduos foi extremamente forte e o imaginário coletivo resultante passou a aceitar os medicamentos produzidos pela indústria farmacêutica como uma das grandes conquistas da Humanidade.

Associado a esse crescimento, a partir da década de 60, um novo fator vai mudar a prática médica e a estrutura do setor prestador de serviços médicos - o aparecimento de um setor capitalista produtor de material e equipamentos médicos. Como conseqüência, não só a velocidade e a taxa de renovação de novos produtos tiveram grande inflexão, como também existiu a necessidade de se criar novas especialidades e especialistas, que pudessem utilizar os novos equipamentos e atender à demanda dos usuários de forma eficiente (Vianna, 1995b).

Isto significou que o padrão de acumulação de capital estava condicionado pelos avanços tecnológicos que ocorriam nos setores farmacêuticos e de equipamentos médicos. Para o setor privado, abriu-se enorme janela de oportunidades. Criaram-se várias trajetórias tecnológicas, que podiam ser exploradas com elevado grau de cumulatividade e apropriabilidade. Os retornos eram tão substanciais que a indústria farmacêutica passou a ter uma das mais altas taxas de lucratividade da economia ${ }^{6}$.

O setor prestador de serviços médicos também se adaptou rapidamente aos novos tempos. Novas práticas e especialidades foram abertas. Uma "boa medicina" já não mais podia ser exercida sem o auxílio de equipamentos de última geração e da solicitação de uma grande quantidade de exames complementares. A saúde tornou-se uma mercadoria para ser vendida e comprada, normalmente a um custo elevado.

Para a população em geral, o momento foi também de grande transformação. A segunda metade do século XX foi a do império da tecnologia ${ }^{7}$.

“(...) o processo de industrialização não pode mais ser compreendido apenas a partir da concentração da indústria, nem mediante uma explicação sobre os 
mecanismos de racionalização do trabalho, mas sim como resultado de uma sociedade organizada para a produção científica e técnica. Na medida em que se insere no interior de cada sociedade, essa racionalidade aparece ao mesmo tempo como fonte de dominação e realização do homem e do Estado" (Guimarães e Vianna, 1994, p. 119).

No imaginário coletivo, a tecnologia está não apenas vinculada ao setor saúde, mas passa a ser um objeto de desejo. Todos, ou praticamente todos, buscam com avidez o último modelo de TV, de aparelho de som, de tomógrafo computadorizado etc. No horizonte, a redenção da Humanidade por meio do desenvolvimento científico-tecnológico parece à vista.

"A ciência já se julga capaz e suficientemente forte para suplantar e substituir todas as demais formações culturais: mitos religiosos. Ideologias metafísicas, saberes estéticos etc. (...) A ciência suspende a dúvida, promete felicidade, assegura que todos o problemas e todas as soluções nascem dela e passam por ela. Trata-se de um fato social total. Nenhuma questão nova - de economia, de prática, de moral, de cultura ou de política - escapa totalmente ao campo de influência da racionalidade científica" (Japiassu, 1985, p. 133).

Na saúde, a tecnologia assume um papel simbólico importante. Ela é o conforto e a garantia de que o conhecimento humano foi utilizado no máximo do seu potencial atual na solução dos problemas individuais. O público demanda, exige, que a prática médica seja calcada na "melhor" tecnologia disponível.

O resultado foi que, devido ao crescente aumento da competição industrial, tanto na área farmacêutica como na de equipamentos, a manutenção das taxas de retorno dos investimentos realizados dependia de um uso extensivo da tecnologia e, portanto, de um aumento crescente dos custos da prática médica. Constrói-se, assim, um cenário explosivo - de um lado, um crescimento vertiginoso da oferta; de outro, uma forte pressão de demanda - cujo resultado é evidente. Os gastos com a saúde aumentam de forma exponencial em praticamente todos os países.

Os gastos com os cuidados de saúde vêm crescendo significativamente em todas as nações industrializadas, particularmente nas últimas duas décadas e em proporções bastante superiores ao crescimento de seus respectivos PIB (Anderson e Poulier, 1999). Entre 1960 e 1990, os gastos com saúde mais que dobraram nos EUA e na grande maioria dos países da OECD 
(OECD, 1998 apud Anderson e Poulier, 1999). Ainda que, na década de 90, a tendência de aumento tenha sofrido uma desaceleração (resultado de processos de reforma dos sistemas de saúde que se expressaram em diversas medidas de contenção e racionalização dos custos), os gastos continuam em ascensão e percentagens substanciais das rendas nacionais são anualmente comprometidas com essas despesas, conforme se evidencia na tabela 1 .

O setor público encontra-se também preso a essa espiral. Sem poder controlar o desenvolvimento tecnológico e pressionado pela população, o Estado se põe a reboque de uma lógica que lhe é totalmente externa. Ainda assim, dois modelos básicos se desenvolvem: um americano, de caráter privado, e outro estatal. No primeiro, o Estado tem baixa participação e o mercado é que vai intermediar o processo de compra e venda da mercadoria saúde. No segundo, o Estado procura manter sua capacidade de regulação, estabelecendo regras de incorporação de tecnologia e de formas de prestação de serviços médicos.

O complexo médico-industrial é, pois, resultante de uma etapa particular do desenvolvimento capitalista da área médica, no qual as práticas privadas e capitalistas tornaram-se hegemônicas e determinantes das funções e relações de cada ator no interior do setor saúde. Nesse sentido, esse complexo não está sozinho vis-à-vis outras práticas sociais do Estado e as necessidades de acumulação de capital do restante da economia. Ao contrário, ele depende - em gênero, número e grau - da integração orgânica que consegue estabelecer com o sistema de produção que o delimita. 
Tabela 1 - Gastos de Saúde em Vinte e Nove Países, 1960, 1990 e 1996

\begin{tabular}{|c|c|c|c|c|c|c|}
\hline & \multicolumn{3}{|c|}{$\begin{array}{c}\text { Gastos em Saúde per capita } \\
\text { (US\$) }\end{array}$} & \multicolumn{3}{|c|}{$\begin{array}{l}\text { Gastos em Saúde como } \\
\text { Percentual do PIB }\end{array}$} \\
\hline & 1960 & 1990 & 1997 & 1960 & 1990 & 1997 \\
\hline Austrália & 94 & 1.320 & 1.805 & 4,9 & 8,3 & 8,4 \\
\hline Áustria & 64 & 1.204 & 1.793 & 4,4 & 7,2 & 7,9 \\
\hline Bélgica & 53 & 1.248 & 1.747 & 3,4 & 7,5 & 7,6 \\
\hline Canadá & 103 & 1.696 & 2.095 & 5,5 & 9,2 & 9,0 \\
\hline Rep. Tcheca & $-\mathrm{b}$ & $-\mathrm{b}$ & $904^{\mathrm{a}}$ & 3,0 & 5,4 & 7,0 \\
\hline Dinamarca & 66 & 1.364 & 1.848 & 3,6 & 8,2 & 7,4 \\
\hline Finlândia & 55 & 1.292 & 1.447 & 3,9 & 8,0 & 7,2 \\
\hline França & 72 & 1.539 & 2.051 & 4,2 & 8,9 & 9,6 \\
\hline Alemanha & 68 & 1.279 & 2.339 & 4,8 & 8,7 & 10,4 \\
\hline Grécia & 16 & 386 & 974 & 2,4 & 4,2 & 7,1 \\
\hline Hungria & $-\mathrm{b}$ & $-{ }^{b}$ & $602 \mathrm{a}$ & $-\mathrm{b}$ & 6,1 & 16,5 \\
\hline Islândia & 50 & 1.374 & 2.005 & 3,3 & 7,9 & 8,0 \\
\hline Irlanda & 35 & 759 & 1.324 & 3,8 & 6,7 & 7,0 \\
\hline Itália & 49 & 1.321 & 1.589 & 3,6 & 8,1 & 7,6 \\
\hline Japão & 26 & 1.082 & 1.741 & 3,0 & 6,0 & 7,3 \\
\hline Coréia & $-\mathrm{b}$ & 310 & 587 & $-\mathrm{b}$ & 3,9 & 4,0 \\
\hline Luxemburgo & $-{ }^{b}$ & 1.495 & 2.340 & $-{ }^{b}$ & 6,6 & 7,1 \\
\hline México & $-{ }^{b}$ & 193 & 391 & $-b$ & 3,6 & 4,7 \\
\hline Holanda & 68 & 1.326 & 1.838 & 3,8 & 8,3 & 8,6 \\
\hline Nova Zelândia & 92 & 937 & 1.352 & 4,3 & 7,0 & 7,7 \\
\hline Noruega & 42 & 1.365 & 1.814 & 3,0 & 7,8 & 7,5 \\
\hline Polônia & $-\mathrm{b}$ & $-\mathrm{b}$ & $371 a$ & $-b$ & 4,4 & 5,2 \\
\hline Portugal & $-\mathrm{b}$ & 614 & 1.125 & $-\mathrm{b}$ & 6,5 & 7,8 \\
\hline Espanha & 14 & 815 & 1.168 & 1,5 & 6,9 & 7,4 \\
\hline Suécia & 89 & 1.492 & 1.728 & 4,7 & 8,8 & 8,6 \\
\hline Suíça & 87 & 1.760 & 2.547 & 3,3 & 8,3 & 10,1 \\
\hline Turquia & $-\mathrm{b}$ & 171 & 260 & $-\mathrm{b}$ & 3,6 & 4,0 \\
\hline Reino Unido & 74 & 955 & 1.347 & 3,9 & 6,0 & 6,7 \\
\hline EUA & 149 & 2.799 & 3.925 & 5,2 & 12,6 & 13,5 \\
\hline Média OECD & 66 & 1.286 & 1.728 & 3,8 & 7,2 & 7,5 \\
\hline
\end{tabular}

Fonte: OECD. Health Data 98: a Comparative Analysis of Twenty-nine Countries (apud Anderson e Poulier, 1999).

Obs.: ${ }^{\mathbf{a}}=$ Gastos ajustados usando-se paridade de poder de compra; ${ }^{\mathbf{b}}=$ Não disponível 


\section{Complexo Médico-financeiro}

À medida que essas práticas capitalistas iam amadurecendo no interior dos sistemas de saúde, o descompasso entre receita e despesa se acentuava. De um lado, devido aos gastos de investimentos em P \& D serem elevados ${ }^{8}$, a capacidade de acumulação de capital dependia de um crescente e constante aumento de recursos financeiros de todos os agentes econômicos privados e públicos. De outro, o fluxo de renda das famílias, via Estado ou recursos próprios, não conseguia acompanhar e compatibilizar o crescimento da oferta com a taxa de risco das doenças a ela associada ${ }^{9}$.

A partir do final dos anos 70 e início da década seguinte, a pressão para se aumentar a eficiência dos sistemas de saúde se tornou muito forte ${ }^{10}$. Nos Estados Unidos, o sistema de managed care cresceu dramaticamente (Glied, 1999). Concomitantemente, o maior domínio de idéias econômicas neoclássicas nesse período fez com que emergisse, nos países europeus com sistemas predominantemente públicos de saúde, a necessidade de se criar mecanismos competitivos, por meio de "políticas orientadas para o mercado" (Glasser, 1993).

O capital financeiro público também se encontrava em dificuldades. Os gastos crescentes impunham um peso que já não podia ser coberto por fundos fiscais. Para os países onde predominava essa lógica de financiamento, era necessário realizar reformas que, em grau maior ou menor, adotassem algumas das práticas propostas pelas empresas de managed care.

O resultado foi a criação de um novo e promissor mercado de valorização de capital. O sistema financeiro, como em praticamente todos os quasemercados capitalistas, assumiu a responsabilidade de financiar a oferta e a demanda. $\mathrm{O}$ esquema empregado foi a securitização do risco, em que uma parcela da população assume direta ou indiretamente, via impostos, o financiamento das despesas. $\mathrm{O}$ objetivo era dar respaldo às práticas do complexo médico-industrial que se formara e dominava a dinâmica dos sistemas de saúde.

$\mathrm{O}$ crescimento desse mercado foi muito forte, principalmente onde o complexo médico-industrial seguia um modelo americano. Não como resultado de uma evolução do antigo complexo médico-industrial, mas sim pela expansão de grupos financeiros como uma de suas áreas de acumulação de capital $^{11}$.

Para o capital financeiro ficou evidente que não era possível manter e sustentar a expansão de gastos esperada pelo CMI. Na defesa de sua 
própria rentabilidade, era necessário obter a hegemonia sobre o comportamento dinâmico dos agentes do setor saúde, por meio de uma racionalização direcionada pelos seus interesses ${ }^{12}$.

O capital financeiro tem, no entanto, uma característica importante. Normalmente, ele é avesso ao risco e à incerteza inerentes às atividades que estão sendo financiadas. Isto significa a necessidade de controlar e regular as atividades de produção, evitando imprevistos e competição predatória. Neste quadro, de forma crescente, os mecanismos de acumulação de capital passaram a ser ordenados e regulados pelo capital financeiro.

Uma conseqüência da crescente participação do capital financeiro foi o aumento da importância de práticas associadas ao managed care. Nesse sentido, era fundamental conter os gastos médicos e promover o uso intensivo da tecnologia. Novas práticas de gerenciamento (managed care), como controle orçamentário, captation $^{13}$ e desenvolvimento de empresas de controle dos benefícios farmacêuticos ofertados etc. foram incorporadas, com impactos crescentes sobre a evolução do sistema.

Baker e Brown (1997) relatam que os planos de saúde nos EUA têm sido responsáveis por uma grande parcela da manutenção de práticas médicas conservadoras, com menor difusão tecnológica. Standard e Poor (Standard \& Poor's Industry Surveys, apud Mortimer, 1997), por sua vez, informam que metade do mercado de medicamentos nos EUA já está sendo comandada por empresas de seguro-saúde, o que tem implicado redução das margens de lucro da indústria farmacêutica e um quadro de tensão e conflito com os grandes laboratórios.

$\mathrm{O}$ resultado foi uma mudança significativa na estrutura e no funcionamento do setor saúde, que se estendeu não apenas ao setor privado, mas teve também fortes influências no setor público. Vários países, como EUA, Inglaterra, Holanda, Alemanha, Israel e França, têm adotado práticas comumente associadas às empresas de managed care (Brown, 1998; Von Otter e Saltman, 1992).

"During the past two decades there has been, in the words of one commentator, a 'competition vogue' within the health care systems of industrialised economies. The movement began with the development of managed care in the US, whereby pre-paid groups of care providers (such as Health Maintenance Organisations) competed for subscribers on the basis of offering the best possible care services at the lowest possible insurance premia. Within the public health care systems of Europe, the movement was translated 
into the implementation of market-like, contractual relationships between health care consumers or their agents, service suppliers and the agencies responsible for health care finance, such as governments or independent insurance funds. Irrespective of the precise form of organisation at the national level, the objectives in all cases have been the same-to drive up efficiency, to improve consumer choice and to control the escalating costs of health care" (Whynes e Baines, 2002, p. 112).

Essas influências têm modificado a relação entre o prestador de serviços médicos e os usuários, o sistema de pagamento, mercado de medicamentos e até o próprio processo de desenvolvimento tecnológico do setor.

Assim, o sistema managed care tem reduzido o reembolso de hospitais e médicos, limitado o uso extensivo de novos procedimentos e testes, e mantido sob controle o uso de tecnologias mais complexas nas principais instituições dos EUA. A redução da lucratividade das novas inovações tem reduzido o ritmo com que elas têm sido adotadas e desenvolvidas. Um exemplo foi a menor taxa de adoção da ressonância magnética no estado de Nova York, nos EUA, na década de 90, devido à pressão das companhias de managed care (Baker, 2000; Glied, 1999).

O capital financeiro, público e privado, tem nesse contexto caminhado a passos largos, na tentativa de impor regras que regulem o mercado. Em conseqüência, entra-se numa nova etapa do desenvolvimento capitalista na área da saúde, em que o capital financeiro pretende tornar-se hegemônico, constituindo agora uma etapa que poderia ser denominada de complexo médico-financeiro. 


\section{Conclusão}

Na realidade, não houve ainda uma superação de um complexo pelo outro, mas, ao contrário, um crescente embate pela hegemonia no setor, no qual as características dos dois complexos podem ser resumidas como:

\begin{tabular}{|l|l|l|}
\hline & $\begin{array}{l}\text { Complexo médico- } \\
\text { industrial }\end{array}$ & $\begin{array}{l}\text { Complexo médico- } \\
\text { financeiro }\end{array}$ \\
\hline Tecnologia & Extensiva & Intensiva \\
\hline Gastos & Crescentes & Decrescentes \\
\hline Modelo Administrativo & Planejamento & Managed Care \\
\hline Hegemonia & Indústria & Finanças \\
\hline
\end{tabular}

O setor saúde compreende uma estrutura abrangente e articulada, integrada pelo Estado, pelas instituições públicas e privadas prestadoras de serviços médicos, pela indústria farmacêutica, de equipamentos e de materiais médicos, bem como pelo conjunto de empresas financeiras e nãofinanceiras atuantes no segmento de assistência médica.

Se, por um lado, a diversidade de padrões de avanço tecnológico e sua posterior incorporação ao setor saúde podem ser explicadas pela amplitude de produtos e serviços envolvidos (desde a produção de seringas a tomógrafos computadorizados e proteínas geneticamente manipuladas), por outro, suas diferentes variedades e velocidade de adoção e difusão são também um produto dos diversos, e com freqüência conflitantes, atores e interesses que atuam no complexo antes médico-industrial e, hoje, cada vez mais, médicofinanceiro.

É nesse espaço - de diversidade, complexidade e conflito - que o Estado precisa atuar para regular e gerir as práticas em saúde. As características, os espaços de intervenção e as limitações apontadas precisam, contudo, ser compreendidas e consideradas em quaisquer tentativas de melhor gerir a tecnologia em saúde, seja no que se refere ao processo de desenvolvimento, no controle da incorporação de tecnologias ao sistema de saúde, na regulação da utilização ou ainda na busca de um uso mais racional e eficiente dos recursos disponíveis. 


\section{Referências Bibliográficas}

ANDERSON, G. F.; POULIER, J. P. Health Spending, Acess, and Outcomes: Trends in industrialized Countries. Health Affairs, May/June, p. 178-192, 1999.

BAKER, L.C. Managed Care and Technology Adoption in Health Care: evidence from magnetic resonance imaging. NBER Working Paper Series. Cambridge, MA, n. 8.020, p. 49, 2000.

BAKER, L.C.; BROWN, L. D. The Effect of Managed Care on Health Care Providers. NBER Working Paper Series. Cambridge, MA, n. 5.987, p. 48, 1997.

BROWN, L. D. Exceptionalism as the Rule? U.S. Health Policy Innovation and Cross-National Learning. Journal of Health Politics, Policy, and Law, v. 23, n. 1, p. 35-51, 1998.

CARLTROM, C. T. The Governemt's Role in the Health Care Industry: past, present, and future. Federal Reserve Bank of Clevelant Economic Comentary, Jun, 1, p. 52, 1994.

CORDEIRO, H. A Indústria da Saúde no Brasil. Rio de Janeiro: Graal, 1980.

GLASSER, W. A. The competition vogue and its outcomes. The Lancet, v. 341, p. 805-812, March, 1993.

GLIED S. Managed Care. NBER Working Paper Series. Cambridge, MA, n. 7.205 , p. $56,1999$.

GUIMARÃES, R.; TAVARES, R. (Org.). Saúde e Sociedade no Brasil: anos 80. Rio de Janeiro: Relume Dumará, 1994.

GUIMARÃES, R.; VIANNA, C.M.M. Ciência e Tecnologia em Saúde. Tendências Mundiais. Diagnóstico Global e Estado da Arte no Brasil. In: CONGRESSO onferência Nacional de Ciência e Tecnologia em Saúde, I. 1994, Brasília. Anais... Brasília: Coordenação Geral de Desenvolvimento Científico e Tecnológico do Ministério da Saúde, 1994.

IGLEHART J.K. Medicare and Prescription Drugs. $N$ Engl J Med, v. 344, n. 13, p. 1.010-1.015, March 29, 2001.

JAPIASSU, H. O nascimento e morte das ciências humanas. Rio de Janeiro: Francisco Alves, 1982.

MORTIMER, R. O. Demand for Prescription Drugs: The Effects of Managed Care Pharmacy Benefits. Berkeley, CA: Departament of Economics, 1997.

NEGRI, B.; GIOVANI, D. (Org.). Brasil: radiografia da saúde, Campinas: 
UNICAMP, 2001.

NUSSENZEIG, H.M. Complexidade e Caos. Rio de Janeiro: UFRJ/CPEA, 1999.

OLIVEIRA, P. M. C. Autômatos Celulares. In: NUSSENZEVEIG, M. Complexidade e Caos. Rio de Janeiro: UFRJ/CPEA, 1999.

PEREIRAE. D. Complexos industriais: discussão metodológica e aplicação à economia brasileira. Dissertação (Mestrado em Economia Industrial) Instituto de Economia Industrial, Universidade Federal do Rio de Janeiro, Rio de Janeiro, 1985.

QUINN, K. The Sources and Types of Health Insurance. Boston: Abt Associates Inc., 1998.

TOWN, R.; VISTNES, G. Hospital Competition in HMO Networks. Journal of Health Economics, v. 20, p. 733-753, 2001.

VIANNA, C. M. M. A Indústria de Equipamentos Médicos: uma análise da estrutura e evolução industrial. Rio de Janeiro: IMS;UERJ, 1995b (Série Estudos em Saúde Coletiva, 133).

VIANNA, C. M. M. Complexo Médico-industrial: notas metodológicas. Rio de Janeiro: IMS;UERJ, 1993 (Série Estudos em Saúde Coletiva, 55).

VIANNA, C. M. M. Indústria Farmacêutica: uma análise da estrutura e evolução industrial. Rio de Janeiro: IMS;UERJ, 1995a (Série Estudos em Saúde Coletiva, 130).

VON OTTER, C.; SAltMAn, R. B. Planned Markets and Public Competition: strategic reform in Northern European health systems. Philadephia: Open University Press, 1992.

WHYNES, D. K.; BAINES, D. L. Primary care physicians' attitudes to health care reform in England. Health Policy, v. 60, p. 111-132, 2002.

\section{NOTAS}

1 Professor adjunto do Departamento de Planejamento e Administração em Saúde do IMS/UERJ.

2 Por exemplo, ver Guimarães e Tavares (1994) e, mais recentemente, Negri e Giovanni (2001).

3 Por exemplo, Von Otter e Saltman (1992) e Carlstrom (1994).

4 “(...) a produção e circulação de medicamentos, a organização da prática médica, as formas de intervenção estatal no setor e as práticas concretas de consumo individual (...) a formação de recursos humanos, as articulações da escola médica com a indústria farmacêutica e as relações com o setor de equipamentos médicos" (Cordeiro, 1980, p. 113). 
5 Para um resumo da discussão atual sobre sistemas complexos, ver Nussenzveig (1999).

6 Para os Estados Unidos, enquanto os gastos em saúde cresceram a uma taxa de 5,6\% entre 1998 e 1999, as despesas com medicamentos tiveram um aumento de 16,9\%. Os gastos per capita com medicamentos corresponderam a 9,4\% dos gastos com saúde em 1999, havendo projeção de que, em 2010, representem cerca de 16,9\% dos gastos com saúde para cada americano (Iglehart, 2001)

7 Esse domínio é absoluto pelo menos até o final dos anos 1970. Os movimentos hyppie e, mais tarde, o ambientalista, procuraram denunciar, cada um a seu modo, os malefícios de se ficar preso à máxima utilização da tecnologia. A degradação do meio ambiente e os seus custos, alguns irreversíveis, podem, segundo denunciam os ambientalistas, ameaçar a própria existência da vida humana na Terra.

8 Gastos elevados não estão apenas relacionados a um grande volume de capital, mas, e talvez até mais importante, à velocidade de giro dos recursos investidos.

9 Como o aparecimento de novas tecnologias na saúde afeta de forma significativa as possibilidades de diagnóstico e de tratamento de várias patologias, o risco de se ter ou não uma determinada doença pode crescer. Por exemplo, tecnologias genéticas têm identificado precocemente várias disfunções em indivíduos que no futuro poderão levar ao aparecimento de graves doenças.

10 "The inevitable clash between service-generated demands for new resources and a macroeconomic imperative to reduce spending has generated broad pressure on health institutions to rethink their organizational and delivery patterns" (Saltman e Von Otter, 1992, p. 22).

11 Mais de $70 \%$ dos americanos têm algum tipo de seguro saúde relacionado às empresas de managed care (Quinn, 1998; Glied, 1999).

12 "The ability of managed care plans to selectively contract with health care providers is an important means by which plans use to control costs. Through selective contracting, a managed care plan can credibly threaten to exclude providers such as hospitals, physicians, or pharmacies from its network, and thereby negotiate lower provider prices" (Town e Vistnes, 2001, p. 2).

13 "Under capitation payment, providers receive a fixed periodic payment for each patient they enroll and can earn more by enrolling more patients (if the capitation fee exceeds expected costs). Capitation makes providers face the full financial cost of their patients' service use, which gives them an incentive to reduce utilization" (Glied, 1999, p. 9). 


\section{ABSTRACT}

Health System Structures: from the medical-industrial to the medicalfinancial complex

The concept of a medical-industrial complex has been used since the 1980s in Brazil to highlight the multiple and complex relations that are established both among various players in the health sector and between them and other sectors of the economy. The medical-industrial complex is a product of the health system's history, and particularly that of its evolution. It is a stage in which, due to the need for reproduction of capital investments, private capitalist practices become hegemonic and determinant in each player's functions, roles, and relations within the system itself. The hypothesis discussed in this article is that the health system now has two fundamental attractors shaping the behavior of its agents: technology and the enhancement of capital value. What has come to be known as the medical-industrial complex was constituted for the former, while the medical-financial complex emerged with the latter.

Keywords: Health system; medical-industrial complex; medical technology.

Recebido em: 16/08/2002.

Aprovado em: 02/12/2002. 